\title{
Design of a Wearable Perturbator for Human Knee Impedance Estimation during Gait
}

\author{
Michael R. Tucker, Adrian Moser, Olivier Lambercy, James Sulzer, Roger Gassert \\ Rehabilitation Engineering Lab, ETH Zurich, Switzerland \\ \{mtucker, amoser, olambercy, jasulzer, gassertr\}@ethz.ch
}

\begin{abstract}
Mechanical impedance modulation is the key to natural, stable and efficient human locomotion. An improved understanding of this mechanism is necessary for the development of the next generation of intelligent prosthetic and orthotic devices. This paper documents the design methodologies that were employed to realize a knee perturbator that can experimentally estimate human knee impedance during gait through the application of angular velocity perturbations. The proposed experiment requires a light, transparent, wearable, and remotely actuated device that closely follows the movement of the biological joint. A genetic algorithm was used to design a polycentric hinge whose instantaneous center of rotation is optimized to be kinematically compatible with the human knee. A wafer disc clutch was designed to switch between a high transparency passive mode and a high impedance actuated mode. A remote actuation and transmission scheme was designed to enable high power output perturbations while minimizing the device's mass. Position and torque sensors were designed for device control and to provide data for post-processing and joint impedance estimation. Pending the fabrication and mechanical testing of the device, we expect this knee perturbator to be a valuable tool for experimental investigation of locomotive joint impedance modulation.
\end{abstract}

Keywords-Actuated knee brace, human joint perturbation, impedance estimation, wafer disc clutch, remote actuation

\section{INTRODUCTION}

Humans continually modulate the mechanical impedance of their knees as a function of the activity to be performed. During locomotion, the joint stiffens at the moment of heel contact in order to absorb the impact forces and to avoid buckling. During swing, the knee becomes compliant so as to maintain stability in the event of a perturbance (i.e., stumbling) and to decrease metabolic energy expenditure [1].

The estimation of human knee impedance is of vital interest to researchers involved with the design and control of variable impedance prosthetic and orthotic devices. With respect to prosthesis design, a device whose controlled passive dynamics have been optimized to emulate those observed in able-bodied subjects will likely enjoy improved biomechanical compatibility, energetic expenditure, and mechanical robustness relative to less informed designs [2]-[5]. As for device control, impedance modulation is necessary to provide stable postural control and could improve gait symmetry, which is hypothesized to decrease the likelihood of secondary impairments [6]. Recent literature has presented a flexible and somewhat biomimetic approach to knee prosthesis impedance

This research is supported by the Swiss National Science Foundation through the National Centre of Competence in Research on Robotics. modulation via surface electromyography (EMG) [7], though the robustness of this approach is limited by the variability of electrode placement, by muscle fatigue, and because only superficial muscle activity is recorded. Many of the other documented transfemoral prosthesis control approaches heuristically estimate the parameters of the impedance control law for a number of finite states for each locomotive activity [3], [8], [9]. Manual tuning of the parameters through this strategy becomes a cumbersome task as the number of states and activities increases for each actuated joint. An increased understanding of the relationships between joint impedance, locomotive state and user-intent would improve our ability to intelligently tune and to seamlessly integrate an orthotic or prosthetic device with the human locomotor system.

The practical estimation of impedance through system identification requires the imposition of a velocity perturbation and measurement of the resulting torque [10]. Some groups have proposed to estimate knee impedance without perturbation through regression of kinetic and kinematic data from motion capture of healthy subjects [2], [8], [11], but these estimates only provide a gross impedance measurement over a specified time interval. Furthermore, it is difficult to generalize these estimates from one activity to another.

There have been relatively few perturbation studies of knee impedance presented in the literature [12]-[15]. Since the experimental conditions reported in these studies are far removed from locomotion (i.e. subjects were seated or laying down, muscular cocontraction not present or not controlled, proprioceptive feedback absent), it is not clear that these results are directly translatable to gait. A few studies characterizing reflex responses to knee joint perturbation have been presented [16], [17], however, corresponding estimates of mechanical impedance were omitted. In [18], a neuromuscular model was used to predict locomotive knee stiffness through correlations with kinetic, kinematic, and EMG data recorded during a seated perturbation experiment. However, this approach neglects the effects of the reflex gain modulation and proprioceptive feedback present during gait [19], and thus requires experimental validation.

Direct experimental estimates in literature of either the magnitude or rate-of-change of knee impedance during locomotion have not been found. This is likely due to the challenges inherent to making such measurements. On one hand, such an experiment would require a device to apply relatively high force or velocity perturbations to the joint, while on the other the device must be both transparent and light enough so as to not significantly alter the subjects nominal gait 


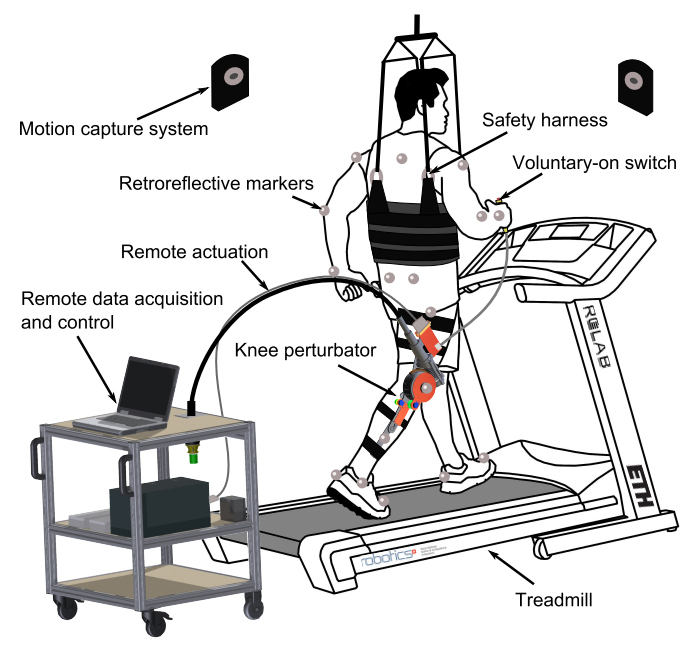

Fig. 1. Rendering of the proposed experimental environment for impedance estimation during level gait.

patterns. The novel device presented in this paper proposes to address these issues and will enable the estimation of dynamic knee impedance during gait. This paper is intended to document some of the design methodologies that were used to develop the knee perturbator and is offered as a reference for the future development of similar devices.

\section{DESIGN REQUIREMENTS}

\section{A. Experimental Design}

The goal of this research is to examine the impedance of the knee through knee velocity perturbations at specific points in the gait cycle with concurrent measurements of reaction torque. In order to accomplish this, it was desired to have a device that would be capable of imposing velocity perturbations to a healthy subject's knee joint during gait within a motion capture environment. A rendering of the envisioned experimental environment for level gait is depicted in Fig. 1. The perturbator must be as transparent as possible such that the subject's nominal (i.e. unperturbed) kinematic and kinetic gait patterns are not fundamentally altered. It was desired to estimate impedance under various locomotive modes (e.g., level walking, stair ascent/descent), and so the device must accomodate different terrain.

A number of alternative perturbation methods were considered for the proposed experiment. Perturbations by a treadmill (e.g., [20]) would be ideal with respect to minimally affecting nominal gait patterns, but would not be capable of perturbation during swing and limits the modes of gait that can be tested. In addition, this type of bottom-up perturbation would not isolate the knee joint and thus may be difficult to analyze. Stationary lower-limb exoskeletons (e.g., [16], [21] were a promising approach since stance and swing could be targeted, but either the transparency during the unperturbed state would be insufficient or the ability to impose velocity (rather than torque) perturbations is limited. Furthermore, these devices would limit the possible modes of gait. Portable lower-limb exoskeletons, especially those which resemble actuated knee braces (e.g., [22]-[24]), appeared to be the best alternative since they could apply single-joint perturbations throughout the
TABLE I. KneE PERTurbator Design ReQuirements

\begin{tabular}{l|c|c}
\hline Specification & Value & Units \\
\hline Range of knee flexion & $0-120$ & $\mathrm{deg}$ \\
Angular perturbation speed & 360 & $\mathrm{deg} / \mathrm{s}$ \\
Maximum torque & 80 & $\mathrm{Nm}$ \\
Device mass & $<3.0$ & $\mathrm{~kg}$ \\
Effect on unperturbed gait & Minimal & - \\
Joint center of rotation & Polycentric & - \\
Enable switches & Voluntary-on & -
\end{tabular}

gait cycle, can accomodate various terrain features, and can be designed specifically for switching between transparency and velocity control modes.

\section{B. Device Design Requirements}

The design requirements are summarized in Table I. The knee flexion angle is constrained from $0-120^{\circ}$ flexion in order to accommodate the full range of motion observed in various locomotive modalities [25]. An average joint perturbation speed of $360^{\circ} / \mathrm{s}$ was chosen to allow for a $10^{\circ}$ perturbation to take place in under $30 \mathrm{~ms}$. Perturbation within this time window is necessary to capture enough data to quantify the prereflexive impedance of the joint [17]. The maximum torque exerted by the brace shall be mechanically limited to $80 \mathrm{Nm}$ so as to avoid injury. While the mass of the device is to be as low as possible, an upper limit of $3.0 \mathrm{~kg}$ was established. In addition, the action of the brace in the absence of perturbations shall have minimal impact on the nominal gait patterns. For this reason, to limit migration of the brace, and for maximum biomechanical compatibility, the brace shall incorporate a hinge that follows the knee joint's instantaneous center of rotation. Finally, it is required for the test subject's safety that the system be enabled by the test subject and the operator through a set of voluntary-on switches.

\section{DeSign Methodology}

The final design of the knee perturbator is depicted in Fig. 2 along with a description of the major components. The rest of this section describes the design methodologies used for selected components.

\section{A. Polycentric Hinge Design}

In order to maximize the kinematic compatibility and to limit the migration of the knee brace relative to the knee, a four-bar linkage was selected based on the findings presented in [26]. Following the methodology presented in that same paper and the models representing human knee joint motion from [27] and [28], a genetic algorithm [29] was used to design a crossed four-bar linkage whose center of rotation (CoR) closely traces the predicted CoR of the biological knee on a vertical plane lateral to the center of the knee. The instantaneous CoR for a crossed four-bar linkage is located at the crossing point of the central linkages. It is straightforward to locate the CoR given the linkage lengths and the input knee angle. Finding the optimal linkage geometry through analytical means is a complex problem, and so the genetic algorithm was used.

The genetic algorithm began with an initial population of individuals - candidate solutions with a randomly generated set of parameters. In this case, each individual solution in the 


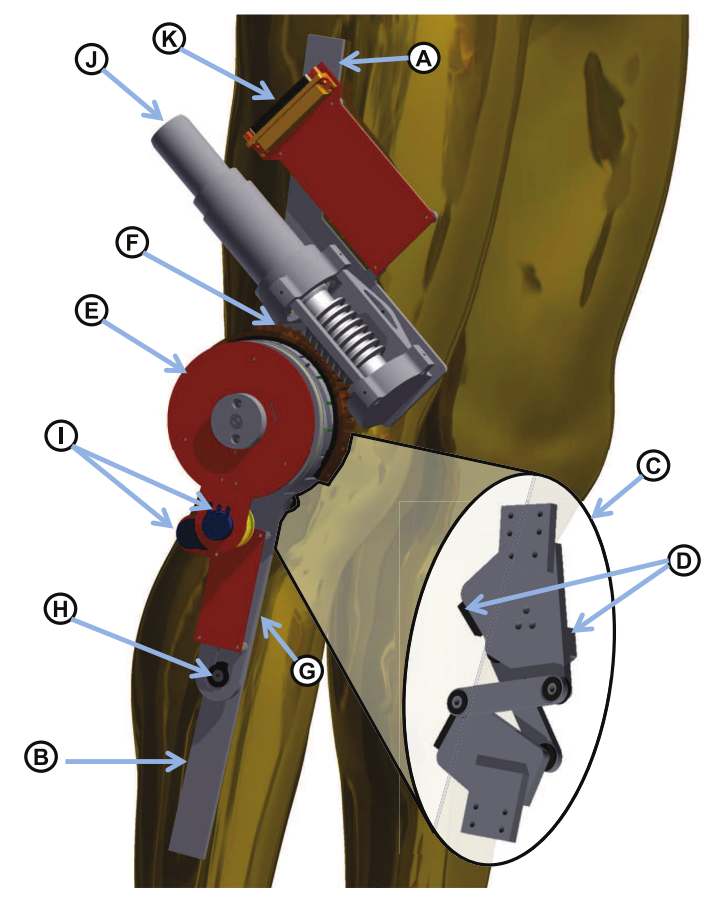

Fig. 2. CAD Rendering of the knee perturbator on a $185 \mathrm{~cm}$ male, shown without guards, straps, or cushioning. Perturbation actuation sequence: femoral link (A) and tibial link (B) are fixed to the subjects right leg. The subject walks normally as four-bar linkage (C) follows the knee joints center of rotation, while safety stops (D) limit the range of flexion from $0-120^{\circ}$. When the wafer disc clutch $(\mathrm{E})$ is engaged, the worm gear $(\mathrm{F})$ mounted on the rotor barrel is coupled to the torque-sensing crank arm $(\mathrm{G})$, thus transmitting power from the femoral link to the tibial link through pin-slider mechanism $(\mathrm{H})$. The knee flexion angle and rotor barrel position are measured using string potentiometers (I) as the flexible shaft (J) drives the knee joint through a velocity perturbation. The position, torque, and other signals are conditioned before being relayed to the control computer via the data acquisition interface $(\mathrm{K})$. The predicted mass of the depicted device is $2.6 \mathrm{~kg}$.

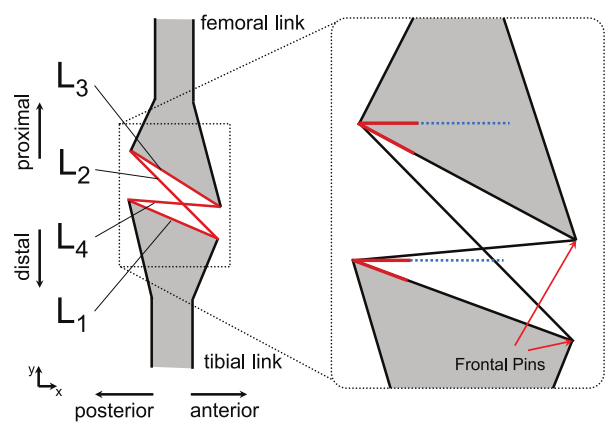

Fig. 3. Geometric parameters used in the genetic algorithm to describe the four-bar linkage.

population was characterized by six parameters representing the physical geometry: four linkage lengths and two initial pitch angles, as shown in Fig. 3. The path of the CoR was then calculated for each individual through an evenly spaced vector spanning the range of motion $\left(0\right.$ to $\left.120^{\circ}\right)$.

The net error between the individual path and the optimal path based on [27] was calculated for each parameter set as a weighted sum of the geometric error in position and the error in the derivative of position (slope error). This was done to penalize solutions whose CoR path was divergent relative
TABLE II. FInAL Four-BAR Linkage PARAMETERS

\begin{tabular}{l|c|c}
\hline Parameter & Value & Units \\
\hline$L_{1}$ linkage length & 44.5 & $\mathrm{~mm}$ \\
$L_{2}$ linkage length & 51.6 & $\mathrm{~mm}$ \\
$L_{3}$ linkage length & 40.9 & $\mathrm{~mm}$ \\
$L_{4}$ linkage length & 47.2 & $\mathrm{~mm}$ \\
$\Psi_{\text {femoral } \text { pitch }}$ & -27.5 & $\mathrm{deg}$ \\
$\Psi_{\text {femoral }}$ pitch & -19.5 & $\mathrm{deg}$
\end{tabular}
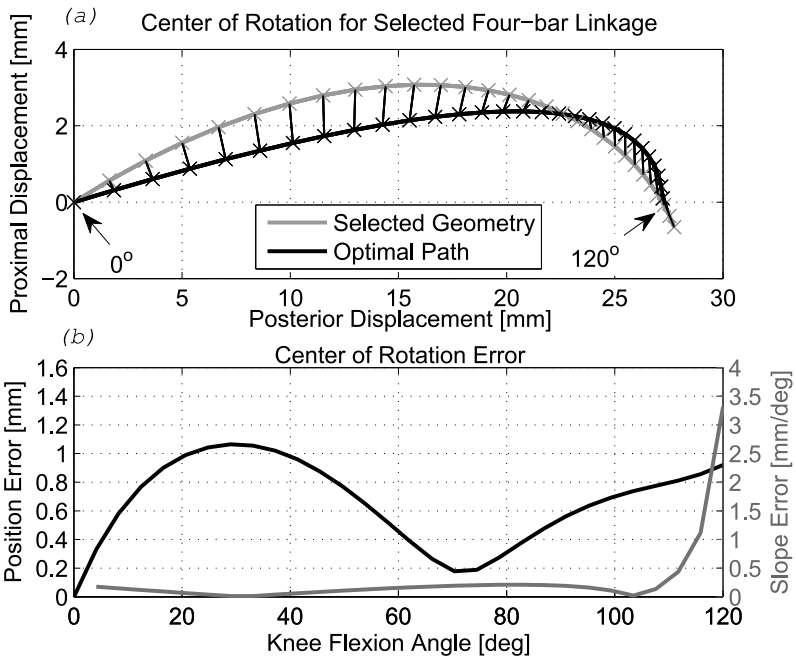

Fig. 4. (a) Path of the center of rotation relative to its initial position at $0^{\circ}$ flexion for the final four-bar linkage with parameters listed in Table II. The optimal path was defined by [27] on a vertical plane $60 \mathrm{~mm}$ lateral to the center of the femoral condyles. Thin black lines connect points with corresponding knee brace angles. (b) Position and slope error for the selected geometry. The maximum position error is $1.06 \mathrm{~mm}$.

to the optimal path despite achieving low positional error. During the cutting phase, checks were performed on whether the parameter set creates infeasible geometry or if it conflicts with another design constraint (e.g., Grashof's condition is satisfied or mechanical interference detected). Any such sets were discarded immediately. For the remaining population, an error threshold was set such that the fittest individuals were kept. The surviving population was then used to create the next generation through direct feed-through, random mutation, and crossover.

The best results obtained through multiple runs of the genetic algorithm are tabulated in Table II along with the CoR trajectory and error in Fig. 4. The maximum position error was $1.06 \mathrm{~mm}$, which is expected to be insignificant given the intersubject anatomical variation and the inherent compliance of the soft tissues to which the device is fixed.

\section{B. Clutch}

One of the most challenging design aspects for the proposed device was the fulfillment of two seemingly contradictory requirements: that the device be capable of imposing a velocity input during perturbation and that the device dynamics are maximally transparent to the knee joint otherwise. The most obvious solution (and that also employed in the perturbator of [22]) was to include a clutch between the driving actuator and the knee brace. The challenge was to find a clutch without backlash that can maintain sufficient 


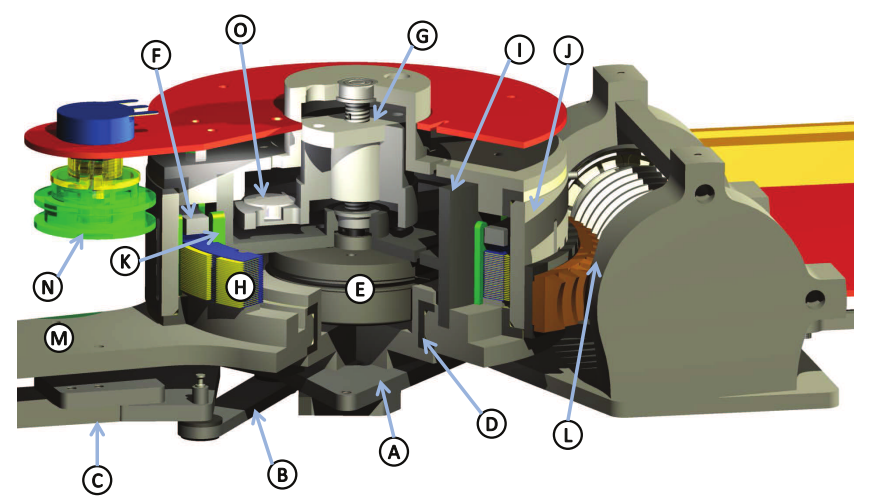

Fig. 5. Cutaway view of the wafer disc clutch based on the brake design of [30]. The wafer disc clutch is mounted on the femoral link (A) just above the four-bar linkage (B) coupling with the tibial link (C). Dry friction bearings (D) on the bottom cover allow the clutch to float relative to the brace. The flat motor $(\mathrm{E})$ actuates the pressure plate $(\mathrm{F})$ through ballscrew mechanism $(\mathrm{G})$, thus applying a normal force to the wafer disc stack $(\mathrm{H})$. The wafer discs are alternately coupled to the stator (I) and the rotor barrels (J) through nylon keys $(\mathrm{K})$. When engaged, the clutch transmits torque from the worm gear $(\mathrm{L})$ to the crank arm (M) and thus to the tibial link. The rotor barrel position is sensed by the string potentiometer $(\mathrm{N})$, while a Hall effect sensor is used to estimate the vertical position of a magnet $(\mathrm{O})$ fixed to the pressure plate.

bidirectional holding torque while being of a reasonable size and weight. Safety considerations, such as maximum torque limitation and automatic release in the event of controller or power failure, imposed additional constraints. An integrated design based strongly on the unlocked version of the multidisc friction brake of [30] was chosen due to its high torque density and intrinsic ability to limit the maximum torque exchanged between the perturbator and the knee.

In the final clutch design, henceforth called the wafer disc clutch, torque is generated by friction resulting from the application of a normal force to a stack of thin wafer discs that are alternately coupled to the stator and rotor tubes of the clutch. The normal force is generated by a pressure plate mounted on a ball screw driven by a flat motor. A moredetailed description and cutaway view of the wafer disc clutch is provided in Fig. 5.

In order to convert the original brake design from [30] to a clutch, an additional degree of freedom was needed. Hence, the rear cover is mounted on dry friction bearings so that the whole assembly can rotate relative to its mount. The stator tube is integrated with the crank arm as one piece, while the worm gear is mounted directly to the rotor tube. The pitch of the ball screw was increased from 1.25 to $1.5 \mathrm{~mm}$ (Thompson NEFF $\mathrm{GmbH}$, Wolfschlugen, Germany) to decrease rise time, with the resulting decreased linear force counteracted by a flat motor with a higher nominal torque (Maxon Motor AG, Sachseln, Switzlerland, EC45 Flat $30 W$ mdl. 339282). Sensing of the pressure plate and rotor barrel positions were also added.

Given the material, geometry and number of the wafer discs, the pitch of the ballscrew, the torque constant of the flat motor, and the current-to-torque relationship given by [30], it was predicted that the static holding torque of the clutch will be $115 \mathrm{Nm}$ at the rated nominal current of the flat motor. Thus, the peak torque limitation of $80 \mathrm{Nm}$ can be realized by either reducing the current to the flat motor or by removing some of the wafer discs. A physical calibration will need to be conducted to validate the intrinsic torque limitation feature.

\section{Actuation}

Due to the relatively high inertia of the lower leg and strength of the knee flexors and extensors, onboard electromagnetic actuation of the perturbator proved to be impractical. Given an example perturbation of $360^{\circ} / \mathrm{s}$ at $40 \mathrm{Nm}$ of torque, this would require about $250 \mathrm{~W}$ of input power. A state-ofthe-art servo motor with $250 \mathrm{~W}$ of power output (e.g., Maxon Motor RE65) alone weighs about $2.1 \mathrm{~kg}$ - about two-thirds of the weight budget for the device.

Thus it was necessary to devise a remote actuation scheme. Design alternatives that were considered included linear and rotational pneumatic and hydraulic actuators, Bowden cables, and flexible drive shafts. Pneumatic and hydraulic pistons were discounted due to the estimated weight and perceived difficulty associated with control and with coupling a linear actuator to a rotational clutch. Rotational pneumatic or hydraulic motors were an appealing option aside from the excessive bulk and weight quoted for commercially available models.

Bowden cables have been demonstrated with some success in lower-limb exoskeletons (e.g. [21]) and in actuated knee braces (e.g. [17], [24]). These provide an extremely lightweight means for applying tensile forces about the center of rotation. However, given the difficulty inherent with implementing coordinated bidirectional position control with Bowden cables and the likelihood of having to repeatedly replace the cables under such high loads and wear conditions, this solution was ultimately not chosen.

For the final design, a custom-made $3.0 \mathrm{~m}$ x $10 \mathrm{~mm}$ bidirectional flexible drive shaft (Haspa $\mathrm{GmbH}$, Ittlingen, Germany) was selected. The shaft length was chosen to give the subject an adequate workspace in the motion capture environment and to maximize the cable's radius of curvature. In order to minimize the weight per unit length of the shaft, to minimize the effects of rotational deflection, and to leverage its affinity for high rotational speed at low torque, we chose to fit the perturbator-end of the shaft with a worm gear set that gives a 60:1 speed reduction. With this configuration, the predicted mass of the perturbator is $2.6 \mathrm{~kg}$, which leaves a manageable $0.4 \mathrm{~kg}$ to account for uncertainties in mass prediction, safety guards, straps and cushioning for fixation.

As a consequence of the four-bar linkage and the pin-slider mechanism, the perturbator has a nonlinear transmission. The net transmission from the flexible shaft to the knee joint is a simple function of the knee angle and is shown graphically in Fig. 6(a). It can be seen that the transmission ratio is roughly $50: 1$ and changes less than $3 \%$ throughout the range of motion. Thus, the drive motor must be capable of delivering at least $0.9 \mathrm{Nm}$ of torque at $3000 \mathrm{rpm}$ in order to meet the perturbation design requirements. In practice, the selected drive motor will need to output substantially more power than this given the inertial dynamics of the perturbator coupled with the leg and the expected losses in the flexible shaft, worm gear, and the friction bearings.

\section{Sensing and Instrumentation}

The two most fundamental quantities to be measured in the proposed experiment are the knee angular velocity and 

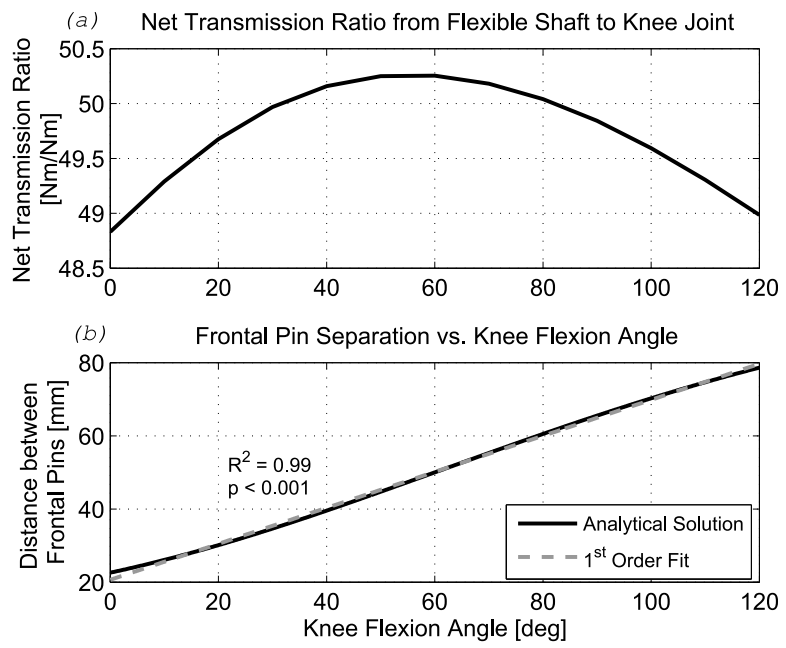

Fig. 6. (a) Net transmission ratio from the flexible shaft to the knee joint as a function of knee flexion angle. (b) Distance between the frontal pins of the four-bar linkage as a function of knee flexion angle. The analytical solution (solid black) is plotted along with a first-order least-squares polynomial fit (dashed grey).

the interface torque between the perturbator and the shank of the leg. The angular velocity is found by differentiating the knee angle with respect to time. Given the geometry of the four-bar linkage and the movement of the center of rotation, direct measurement of the knee angle is nontrivial. While it would be possible to determine this value through geometrical relationships and measurement of the angle between any two linkages, the ranges of motion exhibited at these joints through the full range of knee flexion is small. This small motion would be difficult to resolve using reasonably sized and priced sensors, resulting in a low signal-to-noise ratio upon taking the derivative.

Instead it was decided to measure the linear displacement between the two frontal pins (see Fig. 3) of the linkage since this segment shows the greatest displacement through the movement. To our surprise, the relationship between knee angle and the distance between the frontal pins for this particular geometry is nearly linear, as shown in Fig. 6(b). When the relationship is inverted (i.e. knee angle as a function of frontal pin separation), the maximum angular error is only $3.3 \%$ of the full scale measurement range. As a consequence, the device position can be controlled using a simple linear approximation rather than using the computationally-expensive inversion of the linkage kinematics. Linearity is also desireable from the standpoint of maximizing the net sensor resolution and for signal differentiation.

To measure this displacement, a string potentiometer was chosen due to its compact size and ability to be remotely located. A custom device was designed using an off-the-shelf rotary potentiometer and a constant-force spring. The spring retainer and spool were fabricated on a rapid prototyping machine, with the spool diameter sized such that the full string displacement utilizes the full measurement range of the potentiometer. Based on this design, a second string potentiometer was designed to measure the position of the rotor barrel of the clutch, and can be seen as label (N) in Fig. 5.
The interaction torque is sensed using parallel dual grid strain gages mounted to opposite sides of the crank arm labeled part $(G)$ in Fig. 2. With the gages arranged in a full Wheatstone bridge, the signal is filtered and amplified prior to transmission to the controller. Assuming a bridge excitation $6.0 \mathrm{~V}$ and an amplifier gain of 1000, finite element analysis was used to dimension the crank arm such that a full scale output of $\pm 5 \mathrm{~V}$ is expected at the maximum torque of $80 \mathrm{Nm}$.

\section{CONCLUSIONS AND FUTURE WORK}

The study of human knee impedance modulation during gait has the potential to improve the way in which lower limb prosthetic and orthotic devices are designed and controlled, however it requires the use of a very specialized and high performance tool. A novel lightweight, transparent, remotely actuated, and kinematically compatible knee brace was designed to perform system identification on the joint during locomotion through the application of velocity perturbations and measurement of reaction torques. This device is expected to unlock new experimental paradigms for the study of dynamic postural control.

The design for wafer disc clutch is unique in its predicted torque density and intrinsic maximum torque limiting capability. Such a compact design warrants future consideration in robotic devices that demand rapid switching between a high level of transparency and a rigid coupling with safe power transmission on the scale of human lower-limbs. We propose as future work a catalog and analysis of various clutch topologies (e.g. [23], [30]-[32]) that are specifically appropriate for lowerlimb assistive robotic devices.

While remote actuation has previously been demonstrated in lower-limb exoskeletons [17], [21], [24], the presented device is the first known example that uses a flexible drive shaft. There are a certain number of risks that are assumed while exploring this technology, but the reward potential is sufficient to warrant its use. An experimental characterization of the dynamic properties of the flexible shaft under different bending and loading conditions is planned and is expected to shed light on its feasibility for use in this and other applications.

The knee perturbator is currently being fabricated and will require mechanical testing and verification before it can be attached to a human subject. Additional future work is required to determine the precise experimental conditions and the data post-processing and analysis required for the knee impedance estimation experiment.

\section{ACKNOWLEDGMENT}

The authors would like to thank Pascal Wespe for his numerous contributions toward the design and manufacture of the knee perturbator and Ryan Farris for his personal advice regarding the design of the wafer disc clutch based on his previous work [30].

\section{REFERENCES}

[1] F. Zajac, R. Neptune, and S. Kautz, "Biomechanics and muscle coordination of human walking: Part II: Lessons from dynamical simulations and clinical implications," Gait \& Posture, vol. 17, no. 1, pp. 1-17, 2003. 
[2] E. Martinez-Villalpando, J. Weber, G. Elliott, and H. Herr, "Design of an agonist-antagonist active knee prosthesis," in 2nd IEEE RAS \& EMBS Intl. Conf. Biomedical Robotics and Biomechatronics, 2008. (BioRob '08), 2008, pp. 529-534.

[3] B. Lambrecht and H. Kazerooni, "Design of a semi-active knee prosthesis," in IEEE Intl. Conf. on Robotics and Automation, 2009. (ICRA'09), 2009, pp. 639-645.

[4] M. Arnout, C. Pierre, V. Michael, V. Bram, and L. Dirk, "Concept and design of the HEKTA (Harvest Energy from the Knee and Transfer it to the Ankle) transfemoral prosthesis," in 4th IEEE RAS \& EMBS Intl. Conf. on Biomedical Robotics and Biomechatronics, (BioRob'12), 2012, 2012, pp. 550-555.

[5] R. Unal, R. Carloni, S. Behrens, E. Hekman, S. Stramigioli, and H. Koopman, "Towards a fully passive transfemoral prosthesis for normal walking," in 4th IEEE RAS \& EMBS Intl. Conf on Biomedical Robotics and Biomechatronics (BioRob'12), 2012, 2012, pp. 19491954.

[6] R. Gailey, K. Allen, J. Castles, J. Kucharik, and M. Roeder, "Review of secondary physical conditions associated with lower-limb amputation and long-term prosthesis use," J Rehabil Res Dev, vol. 45, no. 1, p. 15, 2008.

[7] C. D. Hoover, G. D. Fulk, and K. B. Fite, "Stair ascent with a powered transfemoral prosthesis under direct myoelectric control," IEEE/ASME Trans. on Mechatronics, vol. 18, no. 3, pp. 1191-1200, 2013.

[8] F. Sup, A. Bohara, and M. Goldfarb, "Design and control of a powered transfemoral prosthesis," The Intl. Journal of Robotics Research, vol. 27, no. 2, pp. 263-273, 2008.

[9] B. Lawson, A. Huff, and M. Goldfarb, "A preliminary investigation of powered prostheses for improved walking biomechanics in bilateral transfemoral amputees," in 2012 Intl. Conf. IEEE Eng. Med. Biol. Soc. (EMBC'12), 2012, pp. 4164-4167.

[10] R. Kearney, I. Hunter et al., "System identification of human joint dynamics." Crit. Rev. Biomed. Eng., vol. 18, no. 1, p. 55, 1990.

[11] K. Fite, J. Mitchell, F. Sup, and M. Goldfarb, "Design and control of an electrically powered knee prosthesis," in IEEE 10th Intl. Conf. Rehabilitation Robotics, 2007. (ICORR'07), 2007, pp. 902-905.

[12] C. Such, A. Unsworth, V. Wright, and D. Dowson, "Quantitative study of stiffness in the knee joint." Annals of the Rheumatic Diseases, vol. 34, no. 4, pp. 286-291, 1975.

[13] R. Crowninshield, M. Pope, R. Johnson, and R. Miller, "The impedance of the human knee," Journal of Biomechanics, vol. 9, no. 8, pp. 529$535,1976$.

[14] L. Zhang, G. Nuber, J. Butler, M. Bowen, and W. Rymer, "In vivo human knee joint dynamic properties as functions of muscle contraction and joint position," Journal of Biomechanics, vol. 31, no. 1, pp. 71-76, 1997.

[15] C. Tai and C. Robinson, "Knee elasticity influenced by joint angle and perturbation intensity," IEEE Trans. Rehabilitation Engineering, vol. 7, no. 1, pp. 111-115, 1999.

[16] V. Dietz, G. Colombo, and R. Müller, "Single joint perturbation during gait: Neuronal control of movement trajectory," Experimental Brain Research, vol. 158, no. 3, pp. 308-316, 2004.

[17] N. Mrachacz-Kersting, B. Lavoie, J. Andersen, and T. Sinkjaer, "Characterisation of the quadriceps stretch reflex during the transition from swing to stance phase of human walking," Experimental Brain Research, vol. 159, no. 1, pp. 108-122, 2004.
[18] S. Pfeifer, H. Vallery, M. Hardegger, R. Riener, and E. Perreault, "Model-based estimation of knee stiffness," IEEE Trans. Biomedical Engineering, vol. 59, no. 9, pp. 2604-2612, 2012.

[19] V. Dietz, "Proprioception and locomotor disorders," Nature Reviews: Neuroscience, vol. 3, no. 10, pp. 781-790, 2002.

[20] L. Luciani, V. Genovese, V. Monaco, L. Odetti, E. Cattin, and S. Micera, "Design and evaluation of a new mechatronic platform for assessment and prevention of fall risks," J Neuroeng Rehabil, vol. 9, no. 1, p. 51, 2012.

[21] J. Veneman, R. Kruidhof, E. Hekman, R. Ekkelenkamp, E. Van Asseldonk, and H. van der Kooij, "Design and evaluation of the LOPES exoskeleton robot for interactive gait rehabilitation," IEEE Trans. Neural Systems and Rehabilitation Engineering, vol. 15, no. 3, pp. 379-386, 2007.

[22] J. Andersen and T. Sinkjær, "Mobile ankle and knee perturbator," IEEE Trans. on Biomedical Engineering, vol. 50, no. 10, pp. 1208-1211, 2003.

[23] B. Weinberg, J. Nikitczuk, S. Patel, B. Patritti, C. Mavroidis, P. Bonato, and P. Canavan, "Design, control and human testing of an active knee rehabilitation orthotic device," in 2007 IEEE Intl. Conf. on Robotics and Automation, (ICRA'07), 2007, pp. 4126-4133.

[24] J. Sulzer, R. Roiz, M. Peshkin, and J. Patton, "A highly backdrivable, lightweight knee actuator for investigating gait in stroke," IEEE Trans. Robotics, vol. 25, no. 3, pp. 539-548, 2009.

[25] R. Riener, M. Rabuffetti, and C. Frigo, "Stair ascent and descent at different inclinations," Gait \& Posture, vol. 15, no. 1, pp. 32-44, 2002.

[26] J. Bertomeu, J. Lois, R. Guillem, Á. Del Pozo, J. Lacuesta, C. Mollà, P. Luna, and J. Pastor, "Development of a hinge compatible with the kinematics of the knee joint," Prosthetics and Orthotics International, vol. 31, no. 4, pp. 371-383, 2007.

[27] P. Walker, H. Kurosawa, J. Rovick, and R. Zimmerman, "External knee joint design based on normal motion," J Rehabil Res Dev, vol. 22, no. 1, pp. 9-22, 1985.

[28] H. Kurosawa, P. Walker, S. Abe, A. Garg, and T. Hunter, "Geometry and motion of the knee for implant and orthotic design," $J$ Biomech, vol. 18, no. 7, pp. 487-499, 1985.

[29] J. Koza, Genetic programming: A paradigm for genetically breeding populations of computer programs to solve problems. Stanford, CA, USA: Stanford University, Department of Computer Science, 1990.

[30] R. Farris and M. Goldfarb, "Design of a multidisc electromechanical brake," IEEE/ASME Trans. on Mechatronics, vol. 16, no. 6, pp. 985993, 2011.

[31] G. Elliott, "PhD Thesis - Design and evaluation of a quasi-passive robotic knee brace: on the effects of parallel elasticity on human running," Ph.D. dissertation, Massachusetts Institute of Technology, 2012.

[32] M. Tucker and R. Gassert, "Differential-damper topologies for actuators in rehabilitation robotics," in 2012 Intl. Conf. IEEE Eng. Med. Biol. Soc. (EMBC'12), 2012, pp. 3081-3085. 